\title{
Determinación de una mutación en el gen BRCA1 en una familia que presenta cáncer de mama hereditario
}

\author{
Marcela Gallardo $C^{1}$, Paola Faúndez J ${ }^{2}$, Adolfo Cruz, \\ Mario Rodríguez, Manuel Alvarez Z, Pilar Carvallo SQ ${ }^{3}$. \\ Determination of a BRCA1 gene \\ mutation in a family with hereditary \\ breast cancer
}

Background: Breast cancer is the main cause of death among women between 40 and 55 years old, in whom the hereditary cases are common. Therefore, the molecular diagnosis of germ line mutations involved in breast cancer susceptibility is relevant. BRCA1 and BRCA2 have been described as the two major genes involved in familial breast/ovarian cancer. We are performing a screening of BRCA1 and BRCA2 genes, in a group of 50 high risk Chilean families for breast/ovarian cancer. We have detected a mutation, $3936 \mathrm{C}>\mathrm{T}$, that leads to a truncated protein, in two affected women from one of the families in study. Aim: To report the results of the screening for $3936 \mathrm{C}>\mathrm{T}$ in healthy relatives of index women. Material and methods: The molecular diagnosis of this mutation was offered to the healthy members of this family, and 17 relatives accepted to be tested. The region of the BRCA1 gene that includes the $3936 \mathrm{C}>\mathrm{T}$ mutation, was analyzed through PCR amplification, digestion with restriction enzyme BstNI, and direct sequencing. Results: 3936 C>T DNA mutation was present in 8 relatives. Conclusions: Considering the high risk of having a mutation in the BRCA1 gene, specially in pre-menopausal women, the molecualr diagnosis, genetic and clinical counseling are highly relevant. In Chile the molecualr diagnosis is still not widely applied (Rev Méd Chile 2004; 132: 203-8).

(Key Words: BRCA1 protein; Breast neoplasms; Genes, BRCA1)

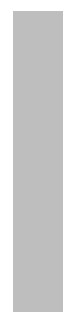

Recibido el 28 de agosto, 2003. Act
Trabajo financiado por el proyecto
Laboratorio de Genética Molecula
Molecular, Facultad de Ciencias
Pontificia Universidad Católica de
Luco-Trudeau.
1Bióloga
2Tecnóloga Médica
3Doctor en Ciencias c/m Biología

Correspondencia a: Dra. Pilar Carvallo de Saint Quentin. Laboratorio de Genética Molecular Humana, Departamento de Biología Celular y Molecular, Facultad de Ciencias Biológicas, Pontificia Universidad Católica de Chile. Casilla 114-D Santiago 7 - Chile. Fax: 56-2 686 2349. E mail: pcarvall@bio.puc.cl 
$\mathrm{E}^{1}$ cáncer mamario es el cáncer de mayor frecuencia en mujeres, representando la segunda causa de mortalidad por cáncer luego del de origen pulmonar, siendo además la primera causa de mortalidad en mujeres entre 40 y 55 años ${ }^{1}$. Por esta razón, esta enfermedad ha sido definida como una de las tres grandes prioridades de atención en las políticas del Ministerio de Salud para la próxima década.

Su incidencia global en países desarrollados ha tenido un continuo crecimiento, cuadruplicándose comparativamente en las dos últimas décadas desde 1 a 1,5\% al año a cifras aproximadas a 4\% de aumento anual ${ }^{2}$. Esta mayor incidencia ha sido particularmente más evidente en mujeres posmenopáusicas. Sin embargo, este significativo aumento en la incidencia, no se ha traducido en un aumento de la mortalidad, la que se habría mantenido estacionaria desde la década 1980-89 y en la que se observan recientes cambios con una tendencia a la declinación ${ }^{3}$.

$\mathrm{Al}$ analizar lo ocurrido, en términos de mortalidad, en grupos etarios específicos, se puede apreciar que un incremento significativo de ésta en mujeres mayores a 55 años ha sido balanceado por una disminución progresiva en mujeres jóvenes ${ }^{4}$. Posiblemente han influido en esta declinación de mortalidad, particularmente observada en pacientes jóvenes, los esfuerzos orientados a mejorar nuestro conocimiento básico de la génesis de esta enfermedad, identificación de factores de riesgo hereditarios $\mathrm{y}$ no hereditarios, mejor sensibilidad $\mathrm{y}$ especificidad de técnicas de detección precoz, generalización en el uso de mamografías como método de análisis poblacional, mayor sensibilización y toma de conciencia a nivel de la población general y de los equipos de salud, y cambios significativos dados por los avances en la terapia.

Existen múltiples factores de riesgo asociados al desarrollo del cáncer de mama, tales como, el sobrepeso, el alcoholismo, la sobredosificación hormonal, las lesiones mamográficas en categoría de alto riesgo y la existencia de familiares cercanos afectados de la enfermedad, siendo este último uno de los de mayor importancia ${ }^{5}$. Se estima que cerca de $5 \%$ de los casos de cáncer de mama son de origen hereditario. Entre los años 1993 y 1995 se identificaron 2 genes de susceptibilidad al cáncer de mama y ovario, el gen BRCA1 localizado en el cromosoma 17q216,7, y el gen
BRCA2, localizado en el cromosoma 13q128,9. El gen BRCA1 está constituido por 24 exones, abarca alrededor de 8,1 $\mathrm{Kb}$ de ADN genómico, su mRNA tiene un tamaño de $7,8 \mathrm{~Kb}$ y codifica para una proteína de 1.863 aminoácidos ${ }^{7}$. Se han realizado varios estudios destinados a entender la función biológica de este gen. Estas investigaciones han revelado que BRCA1 participa en una gran variedad de procesos celulares, entre los cuales figuran la reparación del $\mathrm{ADN}$, la regulación del ciclo celular y la regulación transcripcional de varios genes blanco como p21, GADD45, BAX, PCNA, CFos, CDC34, Ciclina B1 y PIN111-15.

Se han realizado diversos estudios para conocer la frecuencia y la naturaleza de las mutaciones en los genes BRCA1 y BRCA2 en distintas poblaciones del mundo, principalmente en Norteamérica y Europa. De este modo se han descrito hasta el momento más de 1.500 mutaciones para el gen BRCA1 y más de 1.200 para el gen BRCA2, en familias con cáncer de mama hereditario de distintos orígenes étnicos ${ }^{16}$. Estos estudios también establecen que la frecuencia y el tipo de mutaciones en este gen varían considerablemente, dependiendo de la población analizada ${ }^{17}$. Las mutaciones en los genes BRCA1 y BRCA2 confieren un aumento significativo en el riesgo de desarrollar cáncer de mama u ovario ${ }^{18}$, así como un aumento moderado del riesgo de desarrollar otros tipos de cáncer ${ }^{19}$.

Los únicos dos trabajos publicados para este tipo de estudios en población chilena, se refieren al análisis de tres mutaciones específicas. Uno de éstos analiza la presencia de la mutación 185delAG de BRCA1, altamente frecuente en la población judía Ashkenazi, en un grupo de 40 mujeres con cáncer de mama esporádico y 15 mujeres con cáncer de mama familiar ${ }^{20}$. El segundo trabajo, analiza la presencia de la mutación 5382insC en BRCA1 y la mutación 6174delT BRCA2, altamente frecuente en la población judía Ashkenazi, en 382 mujeres sanas con riesgo familiar a desarrollar cáncer de mama, y en 8 mujeres afectadas de cáncer de mama que presentan antecedentes familiares ${ }^{21}$. En ninguno de estos trabajos fue posible detectar la presencia de alguna de estas tres mutaciones.

El diagnóstico molecular de las mutaciones en los genes BRCA1 y BRCA2 es una herramienta tremendamente útil, ya que permite brindar con- 
sejo genético especialmente en aquellos núcleos familiares donde existe una incidencia alta de cáncer de mama y de ovario ${ }^{22}$. Una vez determinada la presencia de alguna mutación en una familia, es posible identificar a los miembros portadores de dicha mutación mediante el diagnóstico molecular presintomático. Los resultados de varios estudios han demostrado que los familiares sanos de personas portadoras de mutaciones en estos genes, tienen un gran interés en someterse a un diagnóstico molecular 23,24 .

Nuestro grupo ha realizado, por los últimos cuatro años, un estudio molecular detallado de los genes BRCA1 y BRCA2 en pacientes chilenas que presentan cáncer de mama u ovario hereditario. En este estudio, que involucra a 50 familias chilenas y que estamos a punto de completar, hemos detectado la presencia de mutaciones en 9 de estas familias ${ }^{25}$. El hallazgo de estas mutaciones y el interés de las pacientes y sus familiares, nos ha llevado a ofrecer el diagnóstico molecular a parientes sanos dentro de cada familia.

En este trabajo presentamos el hallazgo de una mutación en el gen BRCA1 en dos mujeres afectadas de cáncer de mama, y el diagnóstico molecular presintomático que se realizó en 17 familiares sanas, que manifestaron su interés por el estudio.

\section{Material y MÉTodo}

Sujetos. La familia analizada presenta 6 casos de cáncer de mama, 5 de los cuales fueron diagnosticados antes de los 41 años, 2 casos de cáncer de ovario, uno de los cuales fue diagnosticado a los 26 años, y 4 casos de otros tipos de cáncer (Figura 1).

En primer lugar, se analizaron muestras de ADN de 2 mujeres (madre e hija) afectadas de cáncer de mama, mediante secuenciación completa del gen BRCA1. Luego de identificar la mutación en ambas mujeres, se contactó a otros miembros de esta familia y se les ofreció un estudio molecular para determinar la presencia de esta mutación. Se realizó el diagnóstico molecular en 17 mujeres sanas emparentadas en primer grado con las portadoras de la mutación.

Este estudio forma parte de un Proyecto Fondecyt, que ha sido revisado por el Comité de Ética de la Facultad de Medicina de la Pontificia Universidad Católica de Chile. Todas las personas incluidas en el estudio han firmado un documento de consentimiento informado, antes de la toma de muestra y del estudio molecular.

Técnicas

- Obtención de ADN genómico: El ADN genómico se obtuvo a partir de aproximadamente 5

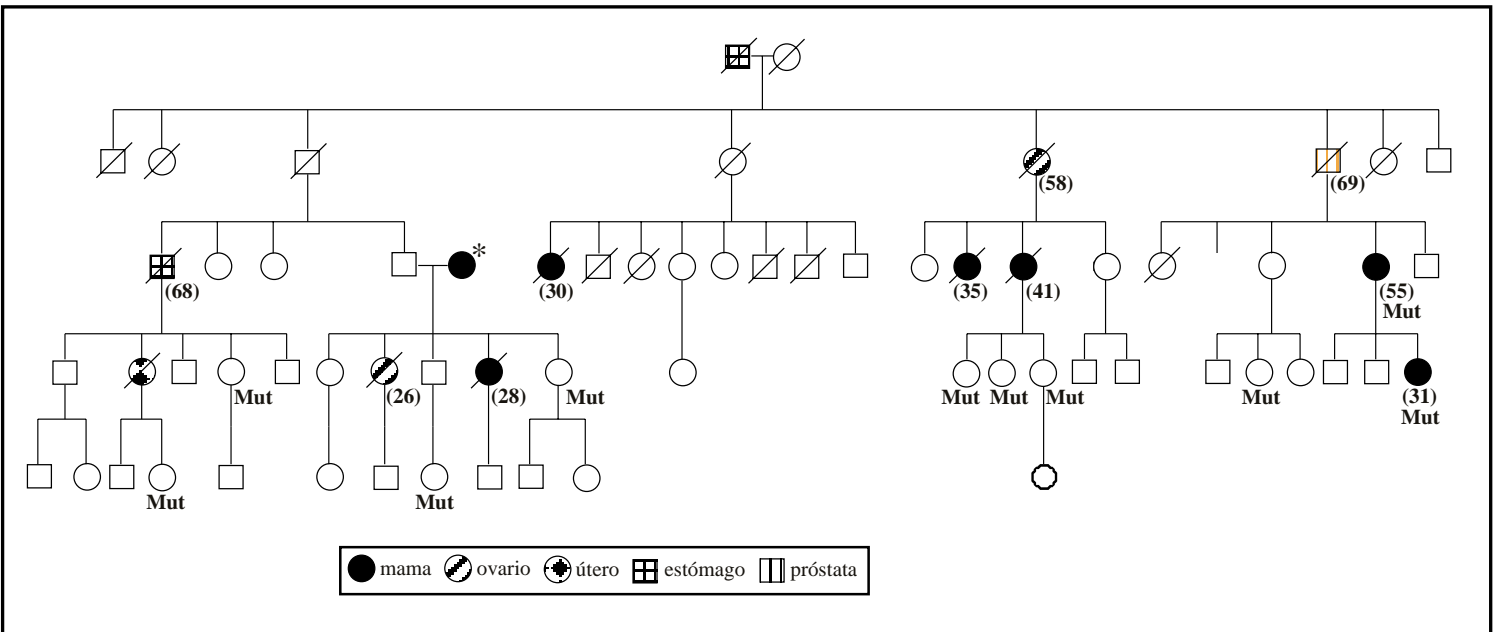

Figura 1. Genealogía de la familia portadora de la mutación 3936 C-T. Los casos de cáncer aparecen señalados con diferentes símbolos y entre paréntesis se indica la edad de diagnóstico. El asterisco indica a una mujer afectada de cáncer de ovario que pertenece a otro linaje. Mut: presencia de la mutación 3936 C>T. 
$\mathrm{ml}$ de sangre venosa, y utilizando el método descrito por Lahiri y Nurnberger ${ }^{26}$.

- La amplificación por PCR de la región carboxilo terminal del exón 11 del gen BRCA1 se realizó utilizando partidores específicos cuya secuencias es:

Partidor sentido:

5'-CGT TGC TAC CGA GTG TCT GTC TAA G-3'

Partidor antisentido:

5'-AGC CCG TTC CTC TTT CTT CAT C-3'

La reacción de amplificación se realizó en un volumen final de $50 \mu \mathrm{l}$, que contiene 25 pmoles de cada partidor, 1 unidad de Taq ADN polimerasa, el tampón suministrado con la enzima ( $\mathrm{KCl} 50 \mathrm{mM}$, Tris- $\mathrm{HCl} 100 \mathrm{mM} \mathrm{pH}$ 8,3, $\left.\mathrm{MgCl}_{2} 15 \mathrm{mM}\right)$, los cuatro desoxinucleótidos (200 $\mu \mathrm{M}$ cada uno) y $100 \mathrm{ng}$ de ADN genómico.

- Para el análisis de restricción del fragmento del exón 11 que contiene la mutación, se tomaron 5 $\mu \mathrm{l}$ del producto de PCR, 1 unidad de enzima BstNI, y 0,5 $\mu \mathrm{l}$ de tampón específico. Esta mezcla fue incubada durante $120 \mathrm{~min}$ a $60^{\circ} \mathrm{C}$. Finalmente, el producto de la digestión se resolvió en un gel de agarosa 3\% en tampón TBE.

- Secuenciación de ADN

Los productos de PCR fueron analizados por secuenciación de ADN utilizando el sistema comercial «dsDNA Cycle Sequencing System».

\section{REsultados y Discusión}

Una de las mutaciones encontradas en el gen BRCA1, en el estudio que llevamos a cabo, corresponde a una transición de Citosina por Timina en la posición 3936 del cADN. Esta mutación no ha sido descrita anteriormente en ninguna otra población y genera la aparición de un codón de término prematuro en el extremo $5^{\prime}$ del exón 11 del gen BRCA1. Esta mutación fue encontrada en dos mujeres (una madre y su hija) afectadas de cáncer de mama, y que pertenecen a una familia con un elevado número de casos de cáncer. Luego de detectar la mutación 3936 C>T en estas dos mujeres, se contactó a las parientes sanas y en riesgo de estas mujeres, y se les ofreció realizar un diagnóstico molecular con el objetivo de identificar a las portadoras de la mutación.

La mutación 3936 C>T genera la pérdida de un sitio de restricción para la enzima BstNI. Mediante un análisis de restricción, realizado como se describe en Métodos, de la región del exón 11 del gen BRCA1 que contiene esta mutación, se analizaron muestras de ADN de otras 17 mujeres de esta familia (Figura 2). En los casos en que se detectó una mutación por este método, éstas fueron confirmadas mediante secuenciación de ADN de la región del gen específica (Figura 3).

Figura 2. Análisis de restricción para la detección de la mutación 3936 C>T: A. Esquema de los sitios de corte de la enzima BstNI en el alelo normal y el alelo mutado. B. Fotografía del gel de agarosa del análisis de restricción del fragmento que contiene la mutación. La digestión con la enzima BstNI, se realizó como se indica en Métodos. Se muestra el resultado obtenido para algunas de las mujeres estudiadas (M18-M22), C: ADN control normal. El tamaño de cada una de las bandas de restricción se señala al costado derecho de la figura. En gris aparece señalada la banda de 200 pares de bases característica del alelo mutado.

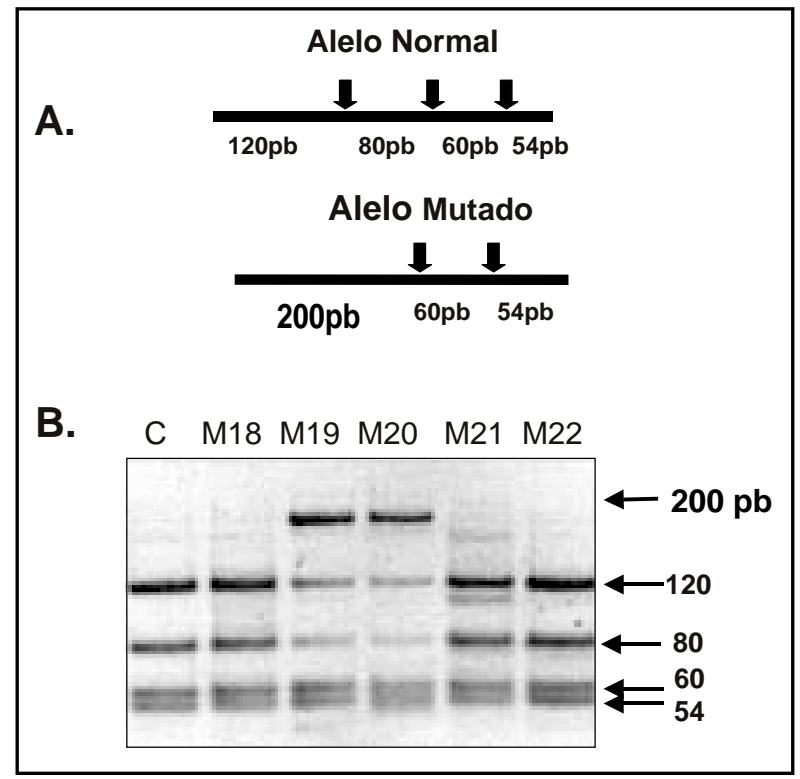




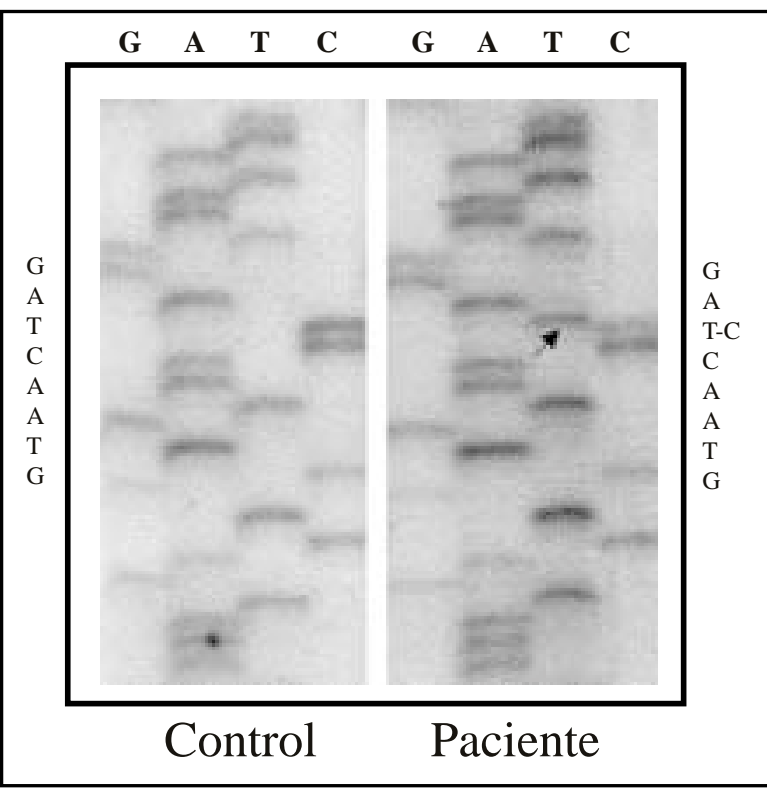

Los resultados muestran que de las 19 mujeres analizadas de esta familia, diez son portadoras de la mutación 3936 C>T en el gen BRCA1. De las 10 portadoras de la mutación, sólo dos han sido diagnosticadas de cáncer de mama, sin embargo, las 8 portadoras restantes corresponden a mujeres jóvenes, con un promedio de edad de 37 años, y que presentan un alto riesgo de desarrollar la enfermedad más adelante.

En esta familia existen 6 casos de cáncer de mama, 5 de los cuales fueron diagnosticados antes de los 41 años, 2 de cáncer de ovario, uno de los cuales fue diagnosticado a los 26 años, 1 de cáncer de útero, 1 de cáncer de próstata y 2 de cáncer de estómago. Es importante destacar que en esta familia existen tres personas que presentaron otro tipo de cáncer, próstata, estómago y útero, y cuyos descendientes presentan la mutación 3936 C>T. Por lo tanto podemos inferir que estas tres personas son portadoras obligadas de esta mutación. Esto es particularmente interesante, ya que en algunas publicaciones se ha relacionado la presencia de mutaciones en el gen BRCA1 con un incremento en el riesgo de desarrollar otros tipos de cáncer, además del cáncer de mama y de ovario. No fue posible analizar la presencia de la mutación 3936 C $>$ T en estos miembros de la familia, ya que todos ellos se encuentran fallecidos, y no ha sido posible obtener las biopsias.
Figura 3. Autorradiografía del gel de secuencia del exón 11: Se secuenció la región del exón 11 del gen BRCA1 en todas las personas que dieron positivo el análisis de restricción. Se muestra una fracción de esta secuencia indicando con una flecha la posición de la transición de Citosina por Timina en uno de los alelos.
En la Figura 1, se señala con un asterisco a una mujer afectada de cáncer de ovario que pertenece a un linaje distinto, y que está casada con uno de los miembros de esta familia. Esta pareja tiene descendencia portadora de la mutación $3936 \mathrm{C}>\mathrm{T}$, que ha sido transmitida por el padre. Además, tienen una hija diagnosticada de cáncer de mama a los 28 años, y una hija diagnosticada de cáncer de ovario a los 26 años, ambas fallecidas antes de los 30 años. Una tercera hija de esta pareja no presenta la mutación 3936 C $>$ T. Sin embargo, es necesario considerar la posibilidad de que en esta rama familiar exista alguna segunda mutación en BRCA1, BRCA2 u otro gen, aportado por la madre. Por lo tanto, no es posible en este caso descartar el alto riesgo de cáncer de mama u ovario en esta mujer. Además, también es importante determinar la presencia de la mutación $3936 \mathrm{C}>\mathrm{T}$ en los hombres de esta familia, ya que aunque ellos no presentan un aumento en el riesgo de desarrollar cáncer de mama, pueden transmitir la mutación a sus hijas.

El diagnóstico molecular de las mutaciones en los genes BRCA1 y BRCA2 es una herramienta tremendamente útil, ya que permite brindar consejo genético, especialmente en aquellos núcleos familiares donde existe una incidencia alta de cáncer de mama y ovario. En estas familias, el diagnóstico molecular permite identificar a los 
individuos portadores de mutaciones, y por lo tanto de mayor riesgo de desarrollar la enfermedad, lo que a su vez puede permitir la aplicación de estrategias preventivas destinadas a reducir este riesgo y a un diagnóstico precoz del cáncer.

Aunque el cáncer de mama hereditario compromete sólo a una pequeña fracción de los casos totales de cáncer de mama, claramente el conocimiento de la presencia o ausencia de mutaciones en los genes BRCA1 y BRCA2 puede tener una importancia clínica significativa. Varios estudios han descrito la existencia de un fenotipo morfológico e inmunohistoquímico particular en tumores mamarios derivados de mujeres portadoras de mutaciones en el gen BRCA1 ${ }^{27-32}$. Estos tumores se caracterizan por ser de alto grado, con un pronunciado pleomorfismo nuclear, y una tasa mitótica excepcionalmente alta ${ }^{30,31,33,34}$, además se caracterizan por ser de tipo medular o medular atípico 32,33 y presentar un crecimiento de tipo trabecular ${ }^{35}$.

Los tumores asociados a mutaciones en BRCA2 parecen tener un fenotipo menos distinguible con respecto al cáncer esporádico, aunque algunos trabajos han informado una alta frecuencia de tumores de alto grado, pero a diferencia de los tumores asociados a BRCA1, presentan una menor tasa mitótica y un crecimiento de tipo acinar ${ }^{30,36}$.

Este fenotipo morfológico característico de los tumores asociados a mutaciones en BRCA1, sugiere un mecanismo molecular distinto de patogénesis en estos casos de cáncer. Estudios inmunohistoquímicos han identificado un inmunofenotipo específico para los tumores asociados a BRCA1, los cuales muestran estabilización de la proteína p53, debido a mutaciones somáticas en este gen 36,37 , además, se caracterizan por la pérdida de expresión de los receptores de estrógeno y progesterona, y comúnmente son ErbB2 negativos ${ }^{27,28,36,38}$. Considerando estos datos, es posible que en pacientes portadoras de mutaciones en estos genes, sea recomendable modificar las estrategias terapéuticas tradicionalmente aceptadas y reemplazarlas por opciones más acordes con las características moleculares de los tumores asociados a BRCA1 y BRCA2.
Las mutaciones en BRCA1 y BRCA2 afectan principalmente a mujeres jóvenes, y las portadoras de mutaciones en estos genes, poseen un elevado riesgo de desarrollar cáncer de mama o de ovario ${ }^{18}$. Además, en mujeres que ya han sido diagnosticadas de cáncer de mama, existe un alto riesgo de desarrollar un cáncer contralateral ${ }^{39-42}$. Por lo tanto, el diagnóstico molecular no sólo es útil en mujeres sanas, sino también en aquellas que ya han sido diagnosticadas con la enfermedad, ya que permite la aplicación de estrategias preventivas para ambos grupos de mujeres. En este sentido, algunos autores recomiendan, para el caso de mujeres afectadas de cáncer de mama, la mastectomía total en vez de la mastectomía profiláctica en la mama no afectada, lo cual reduce en $90 \%$ la incidencia de cáncer ipsilateral y contralateral $^{43,44}$. Por otro lado, en estas mujeres, la ooforectomía es utilizada frecuentemente, ya que reduce en $90 \%$ el riesgo de cáncer de ovario $^{45,46}$, además de reducir en $50 \%$ el riesgo de cáncer de mama contralateral ${ }^{47}$. Por otro lado, la quimioprevención ha sido sugerida como una alternativa a la cirugía profiláctica. La droga más utilizada es el tamoxifeno, la cual ha mostrado reducir en $40 \%$ el riesgo de cáncer contralateral en portadoras de mutaciones afectadas de cáncer de mama ${ }^{48}$, sin embargo, su contribución a la reducción del riesgo en portadoras sanas de mutaciones no ha podido ser demostrada ${ }^{49}$. Tomando en cuenta el alto riesgo que implican las mutaciones en BRCA1 y BRCA2, principalmente en mujeres jóvenes, es importante considerar la incorporación de las mujeres portadoras de mutaciones en estos genes, a protocolos de controles periódicos, quimioprevención y programas de cirugía profiláctica, con el fin de reducir este riesgo.

El diagnóstico molecular está siendo incorporado paulatinamente en la práctica clínica, sin embargo, aún es un proceso en evolución, que necesita la creación de pautas claras con respecto a las implicancias éticas y sicológicas del diagnóstico molecular, la eficacia del tratamiento y las intervenciones profilácticas en portadoras de mutaciones. 


\section{REFERENCIAS}

1. National Center for Health Statistics, SEER cancer statistics review, 1973-1985. Bethesda, MD: US National Cancer Institute, 1998.

2. White E, Lee C, Kristal A. Evaluation of the increase in breast cancer incidence in relation to mammography use. J Natl Cancer Inst 1990; 82: 1546-52.

3. Chu K, TARone R, KesSier L. Recent trends in breast cancer incidence, survival and mortality rates. J Natl Cancer Inst 1996; 88: 1571-9.

4. Chevarley F, Whitw E. Recent trends in breast cancer mortality in black US women. Am J Public Health 1997; 87: 775-81.

5. Garfikel L, Boring C, Heath C. Changing trends: an overview of breast cancer incidence and mortality. Cancer 1998; 74 (suppl): 222-7.

6. HAL JM ET AL. Linkage of early onset familial breast cancer to chromosome 17q21. Science 1990; 250: 1684-9.

7. MIKI Y ET AL. A strong candidate for the breast and ovarian cancer susceptibility gene BRCA1. Science 1994; 266: 66-71.

8. WOOSTER R ET AL. Identification of the breast cancer susceptibility gene BRCA2. Nature 1995; 378: 789-92.

9. Tavtigian SV ET AL. The complete BRCA2 gene and mutations in chromosome 13q-linked kindred. Nat Genet 1996; 12: 333-7.

10. Deng C, Brodie S. Roles of BRCA1 and its interacting proteins. BioEssay 2000; 22: 728-37.

11. WelcSh P, King MC. BRCA1 and BRCA2, and the genetics of breast and ovarian cancer. Hum Mol Genet 2001; 10: 705-13.

12. Venkitaraman A et al. Cancer Susceptibility and functions of BRCA1 and BRCA2. Cell 2002; 108: 171-82.

13. Lu M, Conzen SD, ARRICK BA. Characterization of functional messenger RNA splice variants of BRCA1 expressed in nonmalignant and tumor derived breast cells. Cancer Res 1996; 56: 457881.

14. HARKIN DP ET AL. Induction of GADD45 and JNK/ SAPK dependent apoptosis following inducible expression of BRCA1. Cell 1999; 97: 575-86.

15. MacLachian TK, Somasundaram K et al. BRCA1 effects on the cell cycle and the DNA damage response are linked to altered gene expression. J Biol Chem 2000; 275: 2777-85.
16. Breast Cancer Information Core. http:// research.nhgri.nih.gov/bic/

17. SzaBo CI, KING MC. Population genetics of BRCA1 and BRCA2. Am J Hum Genet 1997; 60: 1013-20.

18. Ford D, Easton DF, Stratton M, Narod S, Goldgar D, Devilee P Et al. Genetic heterogeneity and penetrance analysis of the BRCA1 and BRCA2 genes in breast cancer families. The Breast Cancer Linkage Consortium. Am J Hum Genet 1998; 62: 676-89.

19. FORD D, EASTON DF. The genetics of breast and ovarian cancer. Br J Cancer 1995; 72: 805-12.

20. Trincado P, Fardelia C, Mayerson D, Montero L, Obrien A, Barrueto K, Galuegos R. Prevalencia de la deleción 185delAG del gen BRCA1 en mujeres chilenas con cáncer de mama. Rev Méd Chile 1999; 127: 19-22.

21. Jara L, Ampuero S, Seccia L, Bustamante M, Blanco R, OJEDA JM. Analysis of 5382insC (BRCA1) and 6174delT (BRCA2) mutations in 382 healthy Chilean women with a family history of breast cancer. Biol Res 2002; 35: 85-93.

22. LaLoo F, Evans G. The pathology of familial breast cancer: Clinical and genetic counseling implications of breast cancer pathology. Breast Cancer Research 1999; 1: 48-61.

23. LeRMAn C, NAROd S, Shulman K. BRCA1 testing in families with hereditary breast ovarian cancer: A prospective study of patient decision making and outcomes. JAMA 1996; 275: 1885-92.

24. Lerman C, Seay J, Balshem A, Audrain J. Interest in genetic testing among first degree relatives of breast cancer patients. Am J Med Genet 1995; 57: 385-92.

25. Carvalo P, Palma L, Galardo M, Rousseau C, King MC. Analysis of mutations in the BRCA2 gene in Chilean families with breast cancer. Eur J Hum Genet 2002; 10: 84.

26. LAHIRI DK, NURNBERGER JI. A rapid non-enzymatic method for the preparation of HMW DNA from blood for RFLP studies. Nucleic Acids Res 1991; 19: 5444.

27. Robson M, Rajan P, Rosen PP. BRCA associated breast cancer: absence of a characteristic inmunophenotype. Cancer Res 1998; 58: 1839-42.

28. Lakhani SR, Jacquemier J, Sloane JP. Multifactorial analysis of differences between sporadic cancers and cancers involving BRCA1 and BRCA2 mutations. J Natl Cancer Inst 1998; 90: 1138-45. 
29. KARP SE, TonIN PN, BEgIN LR. Influence of BRCA1 mutations on nuclear grade and estrogen receptor status of breast carcinoma in Ashkenazi Jewish women. Cancer 1997; 80: 435-41.

30. Markus JN, Watson P, Page DL. BRCA2 hereditary breast cancer pathophenotype. Breast Cancer Res Treat 1997; 44: 274-7.

31. Eisinger F, Stoppa-Lyonet D, Longy M. Germ line mutation at BRCA1 affects the histoprognostic grade in hereditary breast cancer. Cancer Res 1996; 56: 471-4.

32. Markus JN, Watson P, Page DL Hereditary breast cancer pathobiology, prognosis, and BRCA1 and BRCA2 gene linkage. Cancer Res 1996; 77: 697-709.

33. Armes JE, Egan AJ, Southey MC. The histologic phenotypes of breast carcinoma occurring before age 40 years in women with and without BRCA1 or BRCA2 germline mutation: a population based study. Cancer 1998; 83: 2335-45.

34. Breast Cancer Linkage Consortium. Pathology of familial breast cancer: differences between breast cancer in carriers of BRCA1 or BRCA2 mutations and sporadic cases. Lancet 1997; 349: 1505-10.

35. ARMES JE, Venter DJ. The pathology of inherited breast cancer. Pathology 2002; 34: 309-14.

36. Armes JE, Trute L, White D. Distinct molecular pathogenesis of early onset breast cancer in BRCA1 and BRCA2 mutation carriers: a population based study. Cancer 1999; 59: 2011-7.

37. Phiшips KA, Nichol K, Ozcelik H. Frequency of p53 mutations on breast carcinomas from Ashkenazi Jewish carriers of BRCA1 mutations. J Natl Cancer Inst 1999; 91: 469-73.

38. Johannsson OT, Idvall I, ANDerson C. Tumour biological features of BRCA1 induced breast and ovarian cancer. Eur J Cancer 1997; 33: 362-71.

39. EASTON D, Ford D, BISHop D. Breast and ovarian cancer incidence in BRCA1 mutation carriers. Am J Hum Genet 1995; 56: 265-71.
40. Robson M, Gilewski T, Hass B. BRCA associated breast cancer in young women. J Clin Oncol 1999; 16: 1642-9.

41. Pierce LJ, Strawderman M, Narod SA. Effect of radiotherapy after breast conserving treatment in women with breast cancer and germline BRCA1/ 2 mutations. J Clin Oncol 2000; 18: 3360-9.

42. Verhoog LC, Brekelmans CT, Seinaeve C. Contralateral breast cancer risk is influenced by the age at onset in BRCA1 associated breast cancer. $\mathrm{Br} \mathrm{J}$ Cancer 2000; 83: 384-6.

43. Hartman LC, Schaid DJ, Woods JE. Efficacy of bilateral prophylactic mastectomy in women with a family history of breast cancer. N Engl J Med 1999; 340: 77-84.

44. Hartman LC, Selers TA, Schaid DJ. Efficacy of bilateral prophylactic mastectomy in BRCA1 and BRCA2 gene mutation carriers. J Natl Cancer Inst 2001; 93: 1633-7.

45. Rebbeck TR, Lynch H, Newhausen S. Prophylactic oophorectomy in carriers of BRCA1 or BRCA2 mutations. N Engl J Med 2002; 346: 1616-22.

46. KaufF N, Satagopan J, Robson M. Risk reducing salpingo oophorectomy in women with a BRCA1 or BRCA2 mutation. N Engl J Med 2002; 346: 1609-15.

47. ReBBeCK TR, LeVIN A, EISEN A. Breast cancer risk after bilateral prophylactic oophorectomy in carriers of BRCA1 or BRCA2 mutations. J Natl Cancer Inst 1999; 91: 1475-9.

48. Narod S, Brunet J, Ghadirian P. Tamoxifen and risk of contalateral breast cancer in BRCA1 and BRCA2 mutation carriers: a case control study. Hereditary Breast Cancer Clinical Study Group. Lancet 2000; 356: 1876-81.

49. King MC, Wieand S, Hale K. Tamoxifen and breast cancer incidence among women with inherited mutations in BRCA1 and BRCA2: National Surgical Adjuvant Breast and Bowel Proyect (NSABPP1) Breast Cancer Prevention Trial. JAMA 2001; 286: 2251-6. 